\title{
A STUDY ON CLINICAL PATTERNS OF VITILIGO AND ITS ASSOCIATED DISEASES IN PATIENTS ATTENDING DERMATOLOGY CLINIC AT A TERTIARY LEVEL CARE CENTRE
}

\author{
Saravanan $N^{1}$, Sindhuja $R^{2}$, Sridhar V3, Rekha G. $P^{4}$, Murugan $S^{5}$ \\ ${ }^{1}$ Associate Professor, Department of Dermatology, Venereology and Leprosy, Chengalpattu Medical College, Tamilnadu. \\ ${ }^{2}$ Assistant Professor, Department of Dermatology, Venereology and Leprosy, Chengalpattu Medical College, Tamilnadu. \\ ${ }^{3}$ Assistant Professor, Department of Dermatology, Venereology and Leprosy, Chengalpattu Medical College, Tamilnadu. \\ 4Junior Resident, Department of Dermatology, Venereology and Leprosy, Chengalpattu Medical College, Tamilnadu. \\ ${ }_{5}^{5}$ Assistant Professor, Department of Dermatology, Venereology and Leprosy, Chengalpattu Medical College, Tamilnadu.
}

\section{ABSTRACT}

\section{BACKGROUND}

Vitiligo is a multifactorial depigmenting condition affecting skin and/or mucous membrane.

The aim of this study is to assess the clinical patterns of vitiligo and its associated diseases in patients attending a tertiary level care centre.

\section{MATERIALS AND METHODS}

This study is a retrospective observational study that includes 200 self-reporting patients of age group 4 - 80 years with vitiligo, who attended Dermatology OPD in Chengalpattu Medical College over a period of one year from October 2015 to September 2016.

\section{RESULTS}

Among various patterns observed, the study obtained the following results overall - vitiligo vulgaris (37\%), focal (19\%), segmental (16\%), mucosal (14\%), acrofacial (8\%), lip-tip (4\%). Among children most common was vitiligo vulgaris (34.4\%) followed by segmental pattern (27.5\%). Most common systemic association was diabetes (18\%) followed by anaemia (14.4\%). Most of them were asymptomatic (85\%) except for the depigmented patches. Most of the associated systemic and cutaneous diseases were found to be common in patients with vitiligo vulgaris than with any other type of vitiligo.

\section{CONCLUSION}

Irrespective of age, sex and duration of disease, the most common pattern is vitiligo vulgaris. Other patterns observed in decreasing order overall are focal, segmental, mucosal, acrofacial, lip-tip, vitiligo universalis with children showing predilection for segmental vitiligo. Most of the patients presented were asymptomatic except for the depigmented patches with the commonest systemic disease association being diabetes followed by anaemia.

\section{KEYWORDS}

Vitiligo, Patterns, Symptoms, Associations.

HOW TO CITE THIS ARTICLE: Saravanan N, Sindhuja R, Sridhar V, et al. A study on clinical patterns of vitiligo and its associated diseases in patients attending dermatology clinic at a tertiary level care centre. J. Evolution Med. Dent. Sci. 2017;6(13):1028-1033, DOI: $10.14260 / \mathrm{Jemds} / 2017 / 220$

\section{BACKGROUND}

Vitiligo is an ancient disease referred by various names "bahak," "kilas," "kodha," "sweta kushta," "sufaid dagh." It is known to mankind by its various patterns since 6000 BC or earlier.[1] It affects $0.5-1 \%$ of the world population ${ }^{[2]}$ with India having an incidence of $0.1-8.8 \%$. The highest incidence has been reported in India followed by Mexico and Japan.[1] It mainly affects younger people with majority of cases presenting before the age of 30 years. Both sexes are equally affected,[2] but many of the studies show a female preponderance ${ }^{[3]}$ because of greater cosmetic awareness and its impact on their social life. [4] The various patterns of vitiligo leads to various cosmetic disfigurements that causes considerable psychological trauma to the patients.

Financial or Other, Competing Interest: None.

Submission 04-01-2017, Peer Review 28-01-2017,

Acceptance 03-02-2017, Published 13-02-2017.

Corresponding Author:

Sindhuja $R$,

Assistant Professor,

Department of Dermatology, Venereology and Leprosy,

Chengalpattu Medical College, GST Road,

Chengalpattu-603001.

E-mail:dhunsi75@gmail.com

DOI: $10.14260 /$ jemds $/ 2017 / 220$

\section{(c) $($ ) $\$$}

\section{MATERIALS AND METHODS}

This study was a retrospective observational study on 200 self-reported patients who attended Dermatology Outpatient Department at Chengalpattu Medical College during the period of October 2015 to September 2016. We enrolled all patients with no exclusions based on sex, nationality, city of residence or socioeconomic status. Details like age, sex, age of onset of lesions, duration and pattern of lesions with associated diseases, blood investigation results like complete blood count, blood sugar levels, thyroid function test, antinuclear antibody (where applicable), urine analysis and audiometric examination results were collected from medical records. Clinical diagnosis of vitiligo was made by detailed history and physical examination.

\section{RESULTS}

Of all the 200 patients included in the study, 91 were male and 109 were female with a male: female ratio of 0.8: 1 and this is in concordance with study made by Sehgal VN, Srivatsava G et al.[1] The age of the patients ranged from $4-80$ years with a mean age of 35.3 years, which is in concordance with study by Krupa Shankar et al.[5] In our study, 66 (33\%) were children $<12$ years which is similar to study made by 
$\mathrm{Hu} \mathrm{Z}$ et al from China.[6] The duration of illness ranged from 3 months to 40 years with mean duration being 3.2 years.

\begin{tabular}{|c|c|}
\hline Sex & Our Study (200 Pts.) \\
\hline Male & $91(45.5 \%)$ \\
\hline Female & $109(54.5 \%)$ \\
\hline M:F Ratio & $0.8: 1$ \\
\hline \multicolumn{2}{|c|}{ Sex Distribution } \\
\hline
\end{tabular}

\begin{tabular}{|c|c|}
\hline Age & $\begin{array}{c}\text { Our Study (200 } \\
\text { Pts.) }\end{array}$ \\
\hline Children (<12 Years) & $66(33 \%)$ \\
\hline Adults & $109(67 \%)$ \\
\hline \multicolumn{2}{|c|}{ Age Distribution } \\
\hline
\end{tabular}

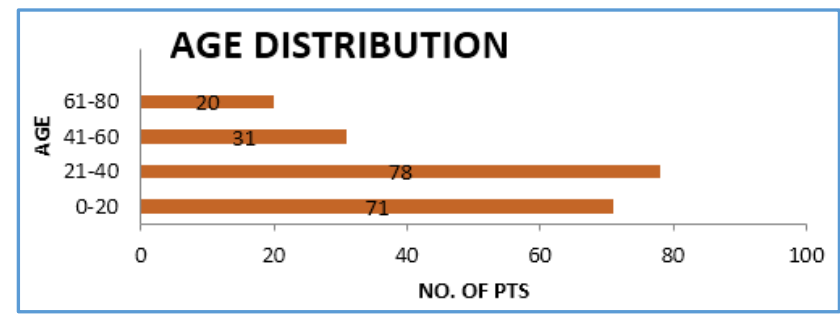

\begin{tabular}{|c|c|}
\hline Parameters (Years) & Our Study (200 Pts.) \\
\hline Age & $4-80$ \\
\hline Mean Age & $35.3 \pm 4.6$ \\
\hline Duration & $0.25-40$ \\
\hline Mean Duration & $3.2 \pm 1.2$ \\
\hline
\end{tabular}

Regardless of the age and duration the most common clinical pattern was vitiligo vulgaris (37\%), which is in concordance with study made by Krupa Shankar et al.[5] It was then followed by focal vitiligo (19\%), acrofacial vitiligo (16\%), mucosal vitiligo (14\%), segmental vitiligo (8\%), lip tip vitiligo $(6 \%)$ and vitiligo universalis $(0 \%)$. Segmental vitiligo was statistically associated with childhood vitiligo (i.e. $60 \%$ of segmental vitiligo were children $<12$ years of age).

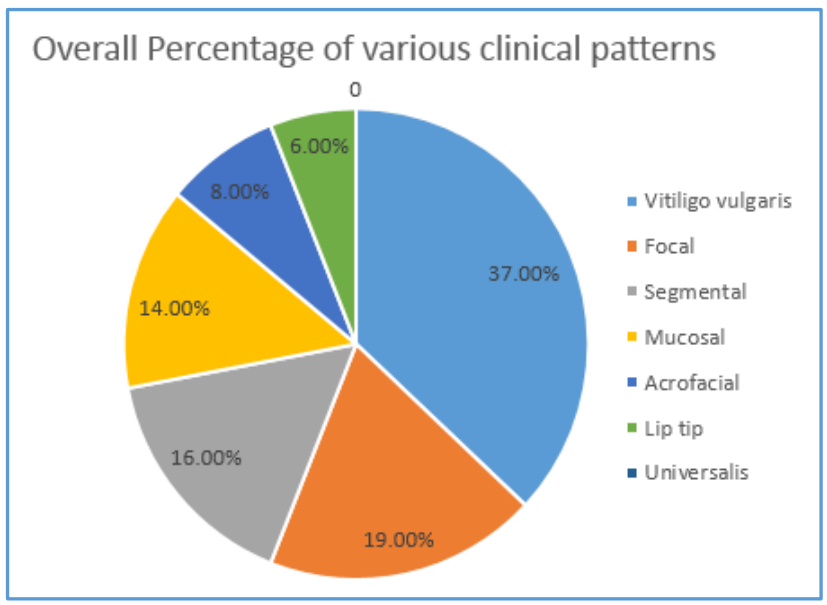

Among children vitiligo vulgaris (34.4\%) was followed by segmental vitiligo (27.5\%), focal (17.2\%), combination vitiligo (10.3\%), lip tip vitiligo (6.8\%) and acrofacial vitiligo (3.4\%). This is in concordance with study made by Palit A et al.[7] Of the 90 patients, 62 (31.2\%) were stable vitiligo and $138(68.8 \%)$ were unstable vitiligo with majority of the stable vitiligo were noticed to be children having early onset and segmental vitiligo.

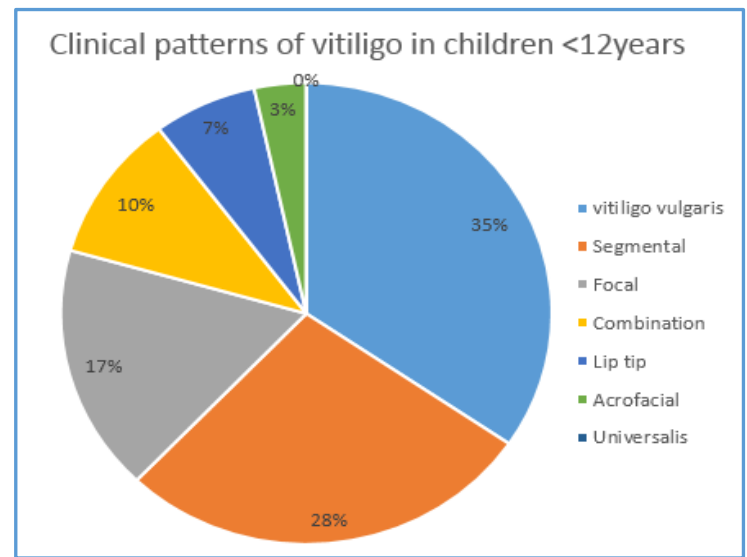

Most of the patients (170 patients - 85\%) were asymptomatic except for the depigmented patches and 20 $(10 \%)$ had pruritus, $6(3 \%)$ had photosensitivity, $4(2 \%)$ had burning sensation. Leukotrichia was found in 36 (18\%) of our patients.

\begin{tabular}{|c|c|}
\hline Symptoms & Out of 200 Pts. \\
\hline Asymptomatic & $170(85 \%)$ \\
\hline Pruritus & $20(10 \%)$ \\
\hline Photosensitivity & $6(3 \%)$ \\
\hline Burning sensation & $4(2 \%)$ \\
\hline
\end{tabular}

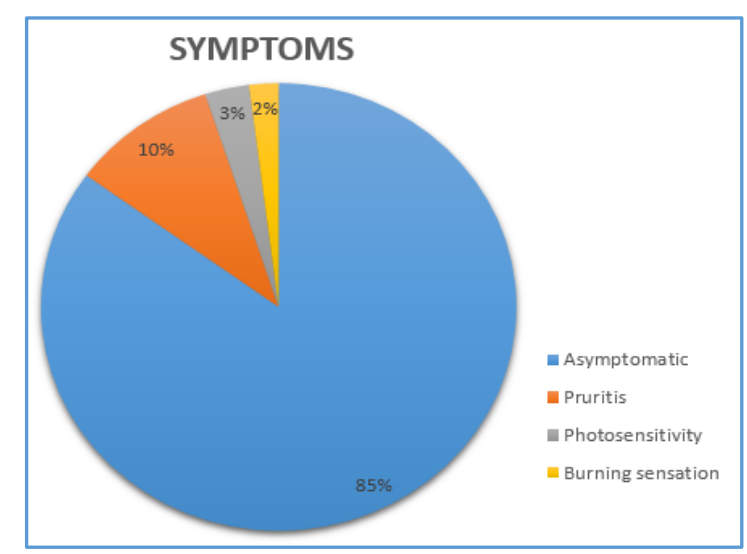

Major associated systemic diseases were found to be diabetes followed by anaemia and thyroid disease. Associated systemic diseases were common in patients with vitiligo vulgaris than with other types of vitiligo and auditory defect was mainly associated in patients with segmental vitiligo. In our OPD cutaneous diseases like psoriasis, lichen planus, dermatophytosis, pruritus, melasma, Hansen's disease and alopecia areata were found to be more commonly associated with vitiligo vulgaris type than with other types of vitiligo.

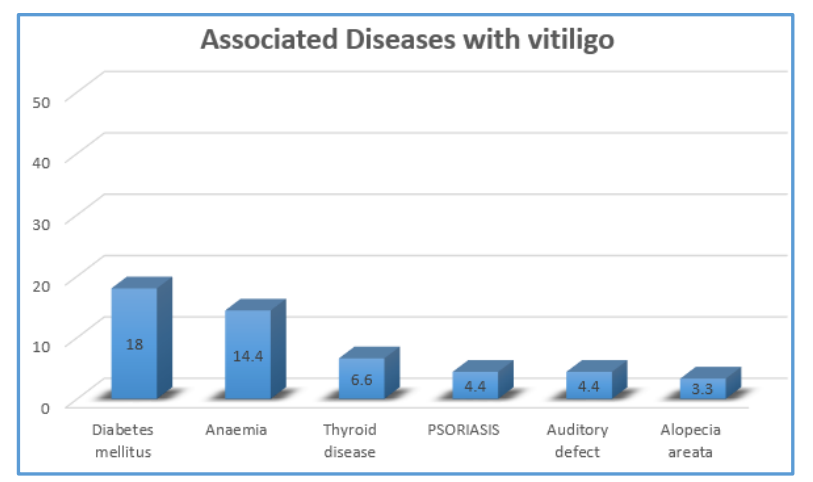




\begin{tabular}{|c|c|c|c|c|c|c|c|}
\hline 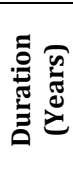 & 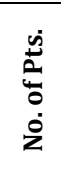 & 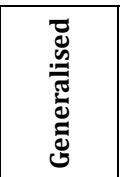 & 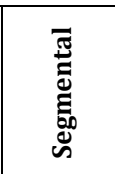 & స్ర్ర & $\begin{array}{l}\bar{\pi} \\
\frac{\pi}{0} \\
\frac{\pi}{0} \\
\frac{0}{4}\end{array}$ & $\begin{array}{l}\bar{J} \\
\stackrel{0}{0} \\
\stackrel{\Xi}{\Sigma}\end{array}$ & $\begin{array}{l}\stackrel{2}{z} \\
\stackrel{\approx}{\exists}\end{array}$ \\
\hline$<1 / 2$ & 45 & \begin{tabular}{|c|}
20 \\
$(\sim 44 \%)$ \\
\end{tabular} & $\begin{array}{c}6 \\
(\sim 13 \%) \\
\end{array}$ & $\begin{array}{c}11 \\
(\sim 24 \%) \\
\end{array}$ & $\begin{array}{c}2 \\
(\sim 4 \%) \\
\end{array}$ & $\begin{array}{c}4 \\
(\sim 8 \%) \\
\end{array}$ & $\begin{array}{c}2 \\
(\sim 4 \%) \\
\end{array}$ \\
\hline $1 / 2-1$ & 42 & \begin{tabular}{|c|}
12 \\
$(\sim 28 \%)$ \\
\end{tabular} & $\begin{array}{c}6 \\
(\sim 14 \%) \\
\end{array}$ & $\begin{array}{c}12 \\
(\sim 28 \%)\end{array}$ & \begin{tabular}{|c|}
2 \\
$(\sim 4 \%)$ \\
\end{tabular} & $\begin{array}{c}7 \\
(\sim 16 \%) \\
\end{array}$ & $\begin{array}{c}3 \\
(\sim 7 \%)\end{array}$ \\
\hline $1-5$ & 81 & \begin{tabular}{|c|}
24 \\
$(\sim 29 \%)$ \\
\end{tabular} & $\begin{array}{c}13 \\
(\sim 16 \%)\end{array}$ & $\begin{array}{c}13 \\
(\sim 16 \%)\end{array}$ & $\begin{array}{c}8 \\
(\sim 9 \%)\end{array}$ & $\begin{array}{c}15 \\
(\sim 18 \%)\end{array}$ & $\begin{array}{c}8 \\
(\sim 9 \%)\end{array}$ \\
\hline$>5$ & 32 & \begin{tabular}{|c|}
8 \\
$(\sim 25 \%)$ \\
\end{tabular} & $\begin{array}{c}6 \\
(\sim 18 \%)\end{array}$ & $\begin{array}{c}2 \\
(\sim 6 \%)\end{array}$ & $\begin{array}{c}6 \\
(\sim 18 \%)\end{array}$ & $\begin{array}{c}6 \\
(\sim 18 \%)\end{array}$ & $\begin{array}{c}4 \\
(\sim 12 \%\end{array}$ \\
\hline Total & 200 & 64 & 31 & 38 & 18 & 32 & 17 \\
\hline
\end{tabular}

Mean Duration - 3.2 yrs. Most common - Generalised vitiligo

\section{DISCUSSION}

Vitiligo is a common, acquired, primary melanocytopenia of unknown aetiology, clinically characterised by well-defined milky white depigmented macules with variable achromotrichia without any change in texture and histologically by degeneration and disappearance of melanocytes in the involved skin, not infrequently in pigment epithelium of the eyes, leptomeninges and inner ear. ${ }^{[8,9]}$

Skin lesions initially presents over the exposed areas and areas with repeated physical trauma like dorsa of hands, feet, elbow and knees in adults.[10] In children it occurs mainly over the face, neck and in infants over diaper and perianal areas. Segmental pattern and achromotrichia have more predilection for children as seen in our study.

The multifactorial disease has various hypothesis on debate of which the currently leading hypothesis is autoimmune theory, [11] which is proved by its association with various autoimmune diseases. The most common among them is thyroiditis. An alteration in the microenvironment of epidermal melanin units, related possibly to immunological and neurochemical factors has been presumed.

\section{Hypothesis Proposed for Vitiligo \\ Autoimmune Theory}

An aberration in immune surveillance is proposed to be the basic event leading to destruction and dysfunction of melanocytes. The theory proposes that a combination of deregulated innate and adaptive immune response are involved in aetiopathogenesis of vitiligo[12,13]; $20-30 \%$ patients have autoimmune endocrinopathies.[14] Primarily, there may be some biochemical trauma to melanocytes leading to release of antigenic substances and subsequent autoimmunisation. Alternatively, there may be autoreactive immune cells directed against the components of autologous melanocytes.

Convincingly, there is frequent presence circulating autoantibodies against thyroglobulin, adrenal, parietal cells, malignant melanoma and halo nevus. It is further supported by the observation of destruction of engrafted melanocytes in a nude mice injected with vitiligo patient's sera.

The evidence of cell-mediated immune defects in vitiligo is supported by the presence of activated CD8+T cells in the perilesional skin. Melanocyte specific T lymphocytes are also detected in peripheral blood of vitiligo patients.[15] There is significant decrease in CD45A+ and significant increase in
HLA-DR+ cells indicating the presence of activated T cells dysregulation. Various associated autoimmune disorders include Hashimoto's thyroiditis, Graves' disease, Diabetes mellitus, Addison's disease, Alopecia areata, Pernicious anaemia, Psoriasis, Inflammatory bowel disease and Autoimmune polyglandular syndrome.

\section{Neural Hypothesis}

This hypothesis is favoured by the common embryological origin of melanocytes and the nervous system and supported by segmental pattern of vitiligo.[16] Evidences are -

1. Occurrence of vitiligo following trauma or damage to nerve.

2. Production of abnormal neuropeptides and nerve growth factors from peripheral nerve endings that are cytotoxic to melanocytes.

3. Abnormal autonomic function such as increased adrenergic tone, increased norepinephrine and catecholamines.

4. Demonstration of direct contact between cutaneous nerve endings and melanocytes in vitiligo macules.

5. Repigmentation in segmental vitiligo following administration of nialamide, an oral MAO inhibitor that inhibits catecholamines.

\section{Self-Destruction Theory by A. B. Lerner}

Intermediate metabolite of melanin synthesis causes melanocyte degradation or the normal process of melanosome degradation proceeds unabated, supported by appearance of vitiligo in areas which are usually darkly pigmented. Tyrosine, DOPA, tryptophan have selective toxicity to melanocytes. Phenolic compounds can cause tyrosinase inhibition. It is supported by the occurrence of vitiligo in normally darkly pigmented areas.[17]

\section{Antioxidant Deficiency Theory}

The antioxidant defense protects the melanocytes from destruction. The reactive oxygen species overproduction leads to accumulation of high 6, 7 biopterins which inhibits tyrosinase activity. Increased oxidative stress increases the process of "haptenation." [18]

\section{Apoptosis}

It can be induced by cytokines or environmental chemicals. There occurs imbalance between pro-apoptotic and antiapoptotic factors. Caspase-3 is the key mediator of melanocyte degradation.[19]

\section{Convergence Theory ${ }^{[20]}$}

It states that there is convergence of genetic factors along with other factors such as stress, autoimmunity, accumulation of toxic substances, impaired melanocyte migration in causation of vitiligo.

\section{New Integrated Theory}

The theory integrates various aetiologies together such as melanocyte detachment, transepidermal loss, neural, biochemical and autoimmune hypothesis. Stress causes indolent detachment and transepidermal loss of melanocytes. Loss of dendricity causes impairment in melanosome transfer further contributing to depigmentation. Melanosomal antigens from damaged melanocytes induces autoimmunity. 


\section{Genetic Influence}

Familial incidence is between $20 \%$ - 30\%. The polygenic inheritance has been suggested. No definite HLA association has been established. Various susceptibility loci are AIS1 (chr1), AIS2 (chr7), AIS3 (chr8), SLEV1 (chr17), VIT 1 GENE on chromosome 2p16 and gene NALP1 on chromosome 17p13. Increased incidence of HLA DR4 in black people, HLA B13 in Moroccan Jews and HLA BW35 in Yemenite Jews[21] have been reported.

\section{Clinical patterns according to:}

\section{Indian Classification}

Classified as segmental vitiligo (Vitiligo zosteriformis) affecting particular dermatomes and non-segmental vitiligo, which includes all others.

\section{Extent of Involvement and Distribution [22]}

1. Segmental Vitiligo (Fig. 3): It is characterised by a unilateral pattern of macules and patches in a dermatomal or quasi dermatomal distribution. This tends to be more common in children with earlier onset and autoimmune disease association. More than 50\% have poliosis (patches of white hair). Alteration in neural peptide is implicated as a cause. Koebnerisation is absent.

2. Generalised Vitiligo or Vitiligo Vulgaris: Most common type and widely symmetrically distributed (Fig. 1).

3. Focal Vitiligo or Vitiligo Areata: An isolated macule or few scattered macules, limited in both size and number (Fig. 2).

4. Acrofacial Vitiligo: It involves the distal digits and periorificial areas of face.

5. Mucosal Vitiligo: It involves oral and genital mucosa, occurs mainly in adults (Fig. 4).

6. Lip-Tip Vitiligo: It involves lips, nipples, distal parts of fingers, toes and penis (Fig. 5).

7. Vitiligo Universalis/Universal Vitiligo: Entire body surface is achromic with few islets of normal or hyperpigmented skin.

8. Combination Vitiligo: Rarely segmental and nonsegmental types occur in same patient.

\section{Morphological Variants Include}

1. Trichrome Vitiligo: An intermediate tan colour that presents as a narrow or broad interface between normally pigmented outer skin and the central depigmented macule.

2. Quadrichrome Vitiligo: This is fourth colour in addition to trichrome vitiligo. There is perifollicular or marginal hyperpigmentation seen in few repigmentation vitiligo cases.

3. Pentachrome Vitiligo: This includes a depigmented macule, tan, brown hyperpigmentation, blue-gray hyperpigmentation and a normally pigmented skin.

4. Inflammatory Vitiligo: Depigmented patches, which has erythematous raised border.

5. Blue Vitiligo: It corresponds to vitiligo occurring in sites of post-inflammatory hyperpigmentation.

6. Confetti Macules: $1-2 \mathrm{~mm}$ in diameter with typical depigmentation, occurs randomly or in a perifollicular manner.
Other variations are hypochromic or vitiligo minor, ${ }^{[2]}$ halo naevi associated vitiligo.

Repigmentation occurs either as diffuse, marginal or perifollicular type. Vitiligo has been associated with various systemic diseases such as diabetes, hypothyroidism, psoriasis, inflammatory bowel disease, hearing and ocular abnormalities and various autoimmune disorders like Hashimoto's thyroiditis, Addison's disease, alopecia areata, pernicious anaemia, SLE and scleroderma.

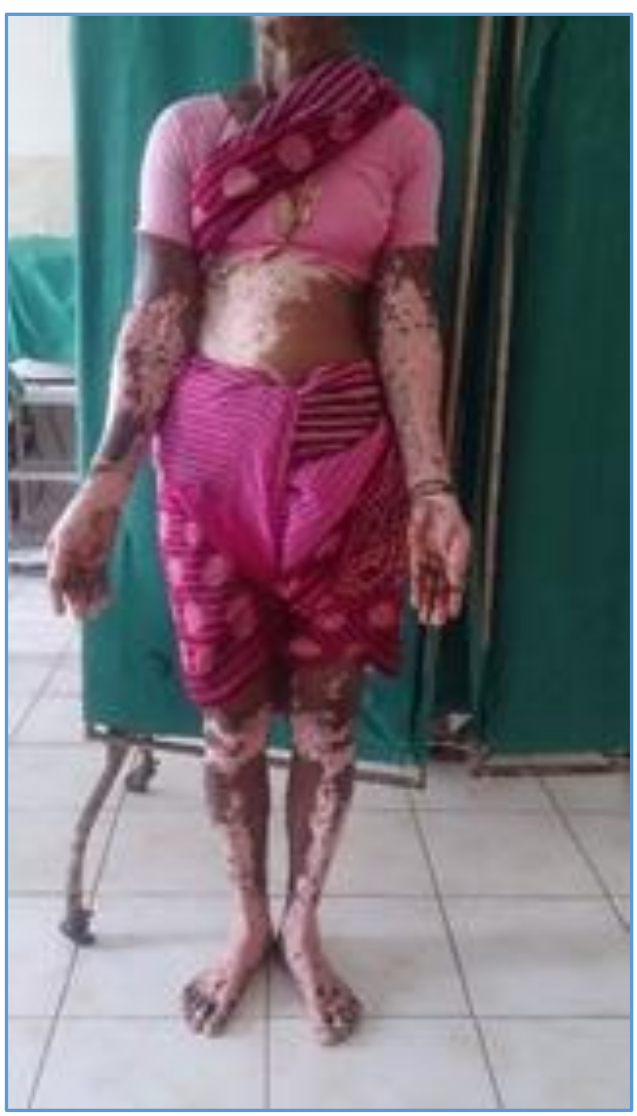

Figure 1. Vitiligo Vulgaris

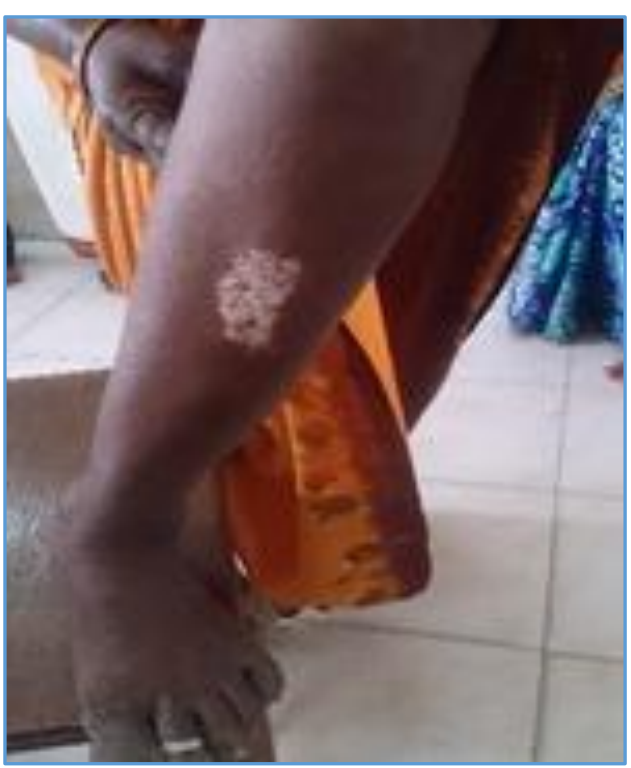

Figure 2. Focal Vitiligo 


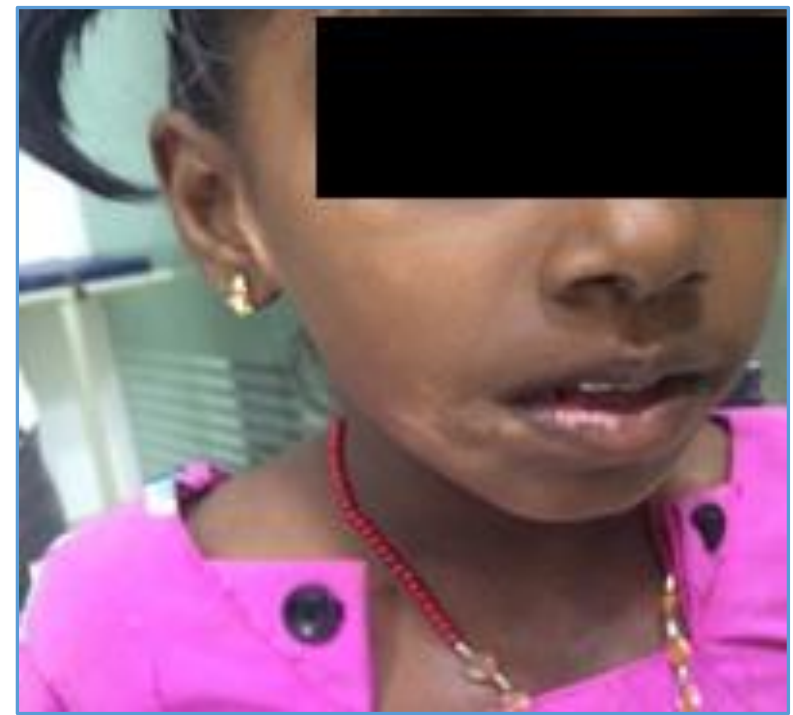

Figure 3. Segmental Vitiligo

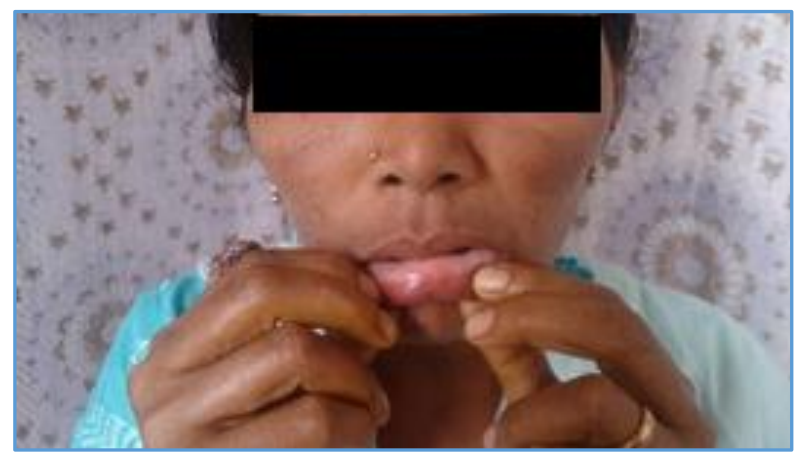

Figure 4. Mucosal Vitiligo

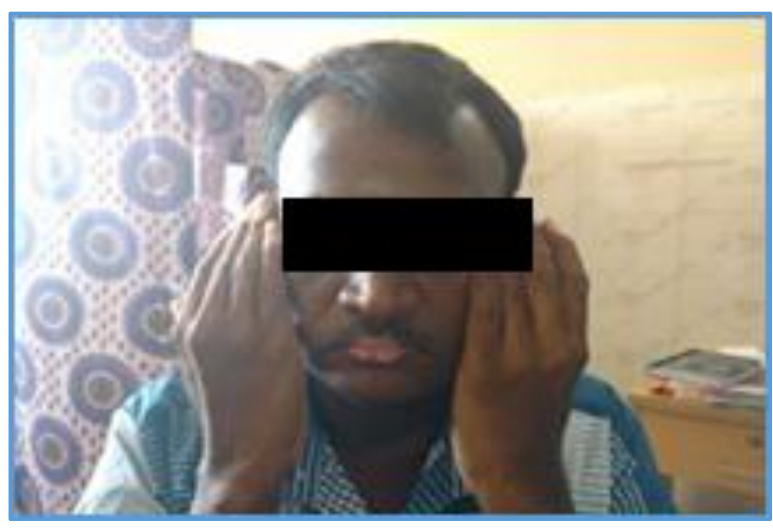

Figure 5. Lip Tip Vitiligo

\section{CONCLUSION}

Vitiligo, although does not affect a person's life expectancy, it is a major psychological trauma to patients. Among various presentations all over the world, our study depicts the clinical patterns and associated diseases in patients attending tertiary level care centre from South India. The most common presentation being generalised vitiligo followed by focal vitiligo and the least common being vitiligo universalis with segmental vitiligo showing increased predilection for children. The commonly associated systemic disease from our centre was diabetes mellitus (mainly above 35 years of age) followed by anaemia. The systemic and cutaneous diseases are most commonly associated with vitiligo vulgaris pattern when compared to any other patterns of vitiligo and regarding cutaneous symptoms most of the patients were asymptomatic except for the depigmented patches.

\section{REFERENCES}

[1] Sehgal VN, Srivatsava G. Vitiligo: compendium of clinico-epidemiological features. Indian J Dermatol Venerol Leprol 2007;73(3):149-56.

[2] Imokawa G. Paracrine interactions of melanocytes in pigmentary disorders. In: Nordlund JJ, Boissy RE, Hearing VJ, et al, eds. The pigmentary system. $2^{\text {nd }}$ edn. Oxford: Blackwell Publishing 2006:421-44.

[3] Howitz J, Brodthagen H, Schwartz M, et al. Prevalence of vitiligo. Epidemiological survey on the isle of Bornholm, Denmark. Arch Dermatol 1977;113(1):47-52.

[4] Martis J, Bhat R, Nandakishore B, et al. A clinical study of vitiligo. Indian J Dermatol Venereol Leprol 2002;68(2):92-3.

[5] Shankar DSK, Shashikala K, Madala R. Clinical patterns of vitiligo and its associated co morbidities: a prospective controlled cross-sectional study in South India. Indian Dermatol Online J 2012;3(2):114-8.

[6] Hu Z, Liu JB, Ma SS, et al. Profile of childhood vitiligo in china: an analysis of 541 patients. Pediatr Dermatol 2006;23(2):114-6.

[7] Palit A, Inamadar AC. Childhood vitiligo. Indian J Dermatol Venerol Leprol 2012;78(1):30-41.

[8] Lerner AB. Vitiligo. J Invest Dermatol 1959;32(2 Pt 2):285-310.

[9] El-Mofty AM. Vitiligo and psoralens. Oxford: Pergamon Press 1968.

[10] Scott G. Rac and rho: the story behind melanocyte dendrite formation. Pigment Cell Res 2002;15(5):322-30.

[11] Hadley ME, Quevedo WC. Vertebrate epidermal melanin unit. Nature 1966;209(5030):1334-5.

[12] Brenner M, Hearing VJ. The protective role of melanin against UV damage in human skin. Photochem Photobiol 2008;84(3):539-49.

[13] Rosdahl I, Rorsman H. An estimate of the melanocyte mass in humans. J Invest Dermatol 1983;81(3):27881.

[14] Dutta AK. Vitiligo: neural and immunologic linkages. 1st edn. Calcutta: Indira Publications 1988.

[15] Mishima Y, Widlan S. Embryonic development of melanocytes in human hair and epidermis. Their cellular differentiation and melanogenic activity. J Invest Dermatol 1966;46(3):263-77.

[16] Boissy RE, Spritz RA. Frontiers and controversies in pathology of vitiligo: separating the wheat from the chaff. Exp Dermatol 2009;18(7);583-5.

[17] Dunn JD, Brady M, Ochsebauer-Jambor C, et al. WNT1 and WNT3a promote expansion of melanocytes through distinct modes of action. Pigment Cell Res 2005;18(3):167-80.

[18] Gilchrest BA, Blog FB, Szabo G. Effects of aging and chronic sun exposure on melanocytes in human skin. J Invest Dermatol 1979;73(2):141-3. 
[19] Kumar R, Prasad D. Melanocytorrhagy and apoptosis in vitiligo: connecting jigsaw pieces. Indian J Dermatol Venerol Leprol 2012;78(1):19-23.

[20] Rosdahl I, Rorsman H. An estimate of melanocyte mass in humans. J Invest Dermatol1983;81(3):27881.
[21] Jin Y, Mailloux CM, Gowan K, et al. NALP1 in vitiligo associated multiple autoimmune disease. $\mathrm{N}$ Engl J Med 2007;356(12):1216-25.

[22] Hann SK, Lee HJ. Segmental vitiligo: clinical findings in 208 patients. J Am Acad Dermatol 1996;35(5 Pt 1):671-4. 